Revista Destaques Acadêmicos, Lajeado, v. 12, n. 4, 2020. ISSN 2176-3070 DOI: http://dx.doi.org/10.22410/issn.2176-3070.v12i4a2020.2367 http://www.univates.br/revistas

\title{
DESEMPENHO TÉRMICO E ACÚSTICO: AVALIAÇÃO DAS PROPOSTAS DE MELHORIAS DE DESEMPENHO TÉRMICO DE UMA EDIFICAÇÃO UNIFAMILIAR MINHA CASA MINHA VIDA
}

\author{
Adriano Inéia ${ }^{1}$, Angélica Ghiggi ${ }^{2}$, Rodrigo Spinelli ${ }^{3}$
}

\begin{abstract}
Resumo: A finalidade deste artigo é a propor uma adaptação de melhoria no desempenho térmico de uma edificação. Sendo esta da categoria residência unifamiliar (Minha Casa minha vida), a proposta consiste em propor um projeto otimizado e a inserção de materiais que favoreçam a minoração da temperatura e favoreça a qualidade de vida nessas habitações, além da economia energética. Ao final, foi possível perceber que as melhorias promoveram o conforto térmico e uma minoração da transmitância térmica. Tudo isso embasado nos conceitos internalizados durante a disciplina de Desempenho Térmico e Acústico, principalmente no quesito de materialidade.
\end{abstract}

Palavras-chave: Desempenho térmico, isolamento térmico e transmitância.

\section{INTRODUÇÃO}

ASHRAE (American Society of Heating, Refrigeration and Air Conditioning Engineers) define que o conforto térmico é o estado da mente que expressa satisfação do homem com o ambiente térmico que o circunda. A insatisfação ou desconforto do usuário, surge da diferença entre o balanço térmico não estável, produzido pelo corpo que é dissipado pelo ambiente, podendo ser a sensação de calor e frio.

O conforto térmico está atrelado a uma série de fatores, sendo elas fisiológica, física e psicológica. Uma obra para promover a qualidade de vida de

1 Engenheiro civil diplomado pela Universidade do Vale do Taquari - UNIVATES. Pósgraduando da Pontifícia Universidade Católica do Rio Grande do Sul PUC RS nos cursos de MBA em Gestão, empreendedorismo e marketing e Pós-graduação em finanças, investimento e banking.

2 Engenheira civil diplomado pela Universidade do Vale do Taquari - UNIVATES.

3 Arquiteto e urbanista diplomado pela Universidade do Vale dos Sinos - UNISINOS. Doutor em Ciência Ambientais, e Docente na Universidade do Vale do Taquari - UNIVATES. 
seus usuários, depende diretamente de um bom isolamento térmico. O cálculo de estimativa do desempenho térmico, parte de dados climáticos, referentes aos dias típicos de projeto (verão/inverno). A estimativa tem como base o projeto arquitetônico, do qual envolve a proporção das trocas que ocorrem entre o ambiente interno e externo. Edificações executadas levando em consideração esses parâmetros, minimizam o consumo de energia elétrica e se tornam mais confortáveis aos usuários.

Uma ressalva importante a se fazer é que cada latitude tem uma carta solar específica. Para o presente artigo, se adotou a latitude $30^{\circ}$ e o período de verão (22 de dezembro), e os valores de temperaturas interna e externas considerados foram $(\mathrm{Ti})=23^{\circ}$ e $(\mathrm{Te})=34^{\circ}$. Portanto, conhecer os materiais e sua resistência quanto à transmissão de calor é fundamental para o melhor dimensionamento do fechamento da estrutura, pois são eles os encarregados pela troca térmica entre o meio interior com o exterior.

A NBR 15220/2003 - Resistência térmica superficial interna e externa determina os valores do (Rse) resistência superficial externa e (Rsi) resistência superficial interna. Estes fechamentos anteriormente citados são responsáveis por receber o calor da radiação e convecção. Uma fração da radiação é absorvida pela superfície e a outra é refletida, isso tudo está correlacionado com os coeficientes de absorvidade $(\alpha)$ e refletividade $(\rho)$ dos materiais. Para maior exatidão os dados empregados no trabalho, foram extraídos dos ábacos e tabelas fornecidos pelo professor.

A temperatura externa quando se elevar acarretará a diferença de temperatura interna que será regida pela condução. Esta ação depende da condutividade térmica do material $(\lambda)$, o mesmo deriva da NBR 15220/2003 - Densidade de massa aparente $(\rho)$, a condutividade térmica $(\lambda)$ e calor específico (c) dos materiais. A proposta do presente trabalho tem como objetivo amadurecer os conhecimentos internalizados sobre desempenho Térmico. Com posse dos coeficientes e da proposta de pesquisa, as dimensões e tipologia de materiais tornam viável o cálculo de resistência de cada seção, conforme a procedência a seguir.

Expressão 01:

$$
R_{\text {Seção }}=\frac{e_{1}}{\lambda_{1}}+\frac{e_{2}}{\lambda_{2}}+\cdots+\frac{e_{n}}{\lambda_{n}}
$$

Onde:

$\mathrm{R}_{\text {seção }}$ resistência térmica da seção analisada.

e: espessura da camada de cada material.

$\lambda$ : condutividade térmica.

A partir dos valores obtidos nas diferentes seções presentes no fechamento analisado é possível calcular a resistência do mesmo, determinada pela expressão. 
Expressão 02:

$R_{t}=\frac{A_{1}}{\frac{A_{1}}{R_{1}}}+\frac{A_{2}}{\frac{A_{2}}{R_{2}}}+\cdots+\frac{A_{n}}{\frac{A_{n}}{R_{n}}}$

Onde:

$\mathrm{R}_{\mathrm{t}}$ : resistência térmica do fechamento analisado.

A: área de cada seção do fechamento.

R: resistência térmica de cada seção do fechamento.

A resistência térmica total da seção é dada pela expressão 3.

Expressão 03:

$R_{T}=R_{s i}+R_{t}+R_{s e}$

Onde:

$\mathrm{R}_{\mathrm{T}}$ : resistência térmica total.

$\mathrm{R}_{\mathrm{t}}$ : resistência térmica do fechamento.

$\mathrm{R}_{\mathrm{si}}$ : resistência térmica superficial interna.

$\mathrm{R}_{\text {se }}$ : resistência térmica superficial externa.

A transmitância térmica do fechamento é o inverso da resistência térmica total, conforme expressão 4.

Expressão 04:

$$
U=\frac{1}{\mathrm{R}_{T}}
$$

\section{Onde:}

U: transmitância térmica.

$\mathrm{R}_{\mathrm{T}}$ : resistência térmica total.

A partir do valor da transmitância é possível calcular o fluxo térmico (qfo) que é dado pela expressão 5 .

Expressão 05

$q_{f o}=U \cdot\left(\alpha \cdot I \cdot R_{s e}+\left(T_{e}-T_{i}\right)\right.$

Onde:

$\mathrm{Q}_{\mathrm{fo}}$ : fluxo térmico.

U: transmitância térmica.

$\alpha$ : absorvidade da superfície externa.

I: radiação solar.

$\mathrm{R}_{\mathrm{se}}$ : resistência térmica superficial externa.

$\mathrm{T}_{\mathrm{e}}$ : temperatura externa. 
$\mathrm{T}_{\mathrm{i}}$ : temperatura interna.

Em seguida é preciso calcular o fluxo térmico total que atravessa o fechamento $(Q)$ que é dado pela expressão 6.

$$
Q=q_{f o} \cdot A
$$

\section{Onde:}

Q: fluxo térmico total que atravessa o fechamento.

$\mathrm{Q}_{\mathrm{fo}}$ : fluxo térmico.

A: área do fechamento.

Primeiramente, se fez um levantamento das normas vigentes de conforto térmico. As normas consultadas foram a NBR 15220 - Norma de desempenho térmico de edificações, a NBR 16401 - Norma de Ar Condicionado e a NBR 15575 - Norma de Desempenho para Edificações Habitacionais, tudo isso visando verificar todas as necessidades, e sanar todas adaptações que assegurem o conforto térmico da edificação em sua totalidade.

\section{PROJETO INICIAL}

O presente trabalho tem como plano de fundo analisar e propor um uma adaptação que maximize o conforto térmico da edificação. $\mathrm{O}$ empreendimento em questão se trata de uma casa unifamiliar da categoria Minha Casa Minha Vida, tendo uma área de $69,85 \mathrm{~m}^{2}$. A análise se iniciou com o cálculo de transmitância térmica de cada ambiente da residência, com posse destes resultados, podemos traçar uma estratégia que desempenhe um bom conforto térmico.

A edificação em questão é térrea e unifamiliar, tendo um pé direito de 2,60m. Possuindo, dois dormitórios, uma cozinha e sala de estar conjugada, um banheiro e um escritório, os parâmetros que norteiam o trabalho, se encontram especificados no Capítulo da 1 Introdução.

As características construtivas adotadas para este estudo são de paredes de $14 \mathrm{~cm}$, constituídas de tijolos cerâmicos seis furos (Figura 01), tendo as duas faces rebocadas e devidamente revestidas com uma pintura cor de pêssego. $O$ forro empregado na edificação é de madeira (pinus), tendo espessura de 1,5 $\mathrm{cm}$ e as telhas adotadas são de barro com espessura de $1 \mathrm{~cm}$. Já as esquadrias da edificação são as denominadas venezianas de madeira e cor de terracota, espessura de $2,5 \mathrm{~cm}$ e os vidros usados são classificados como simples, e espessura de $6 \mathrm{~mm}$. 
Figura 01 - Tijolo cerâmico seis furos (9x14x19 cm), conforme discriminação do fabricante

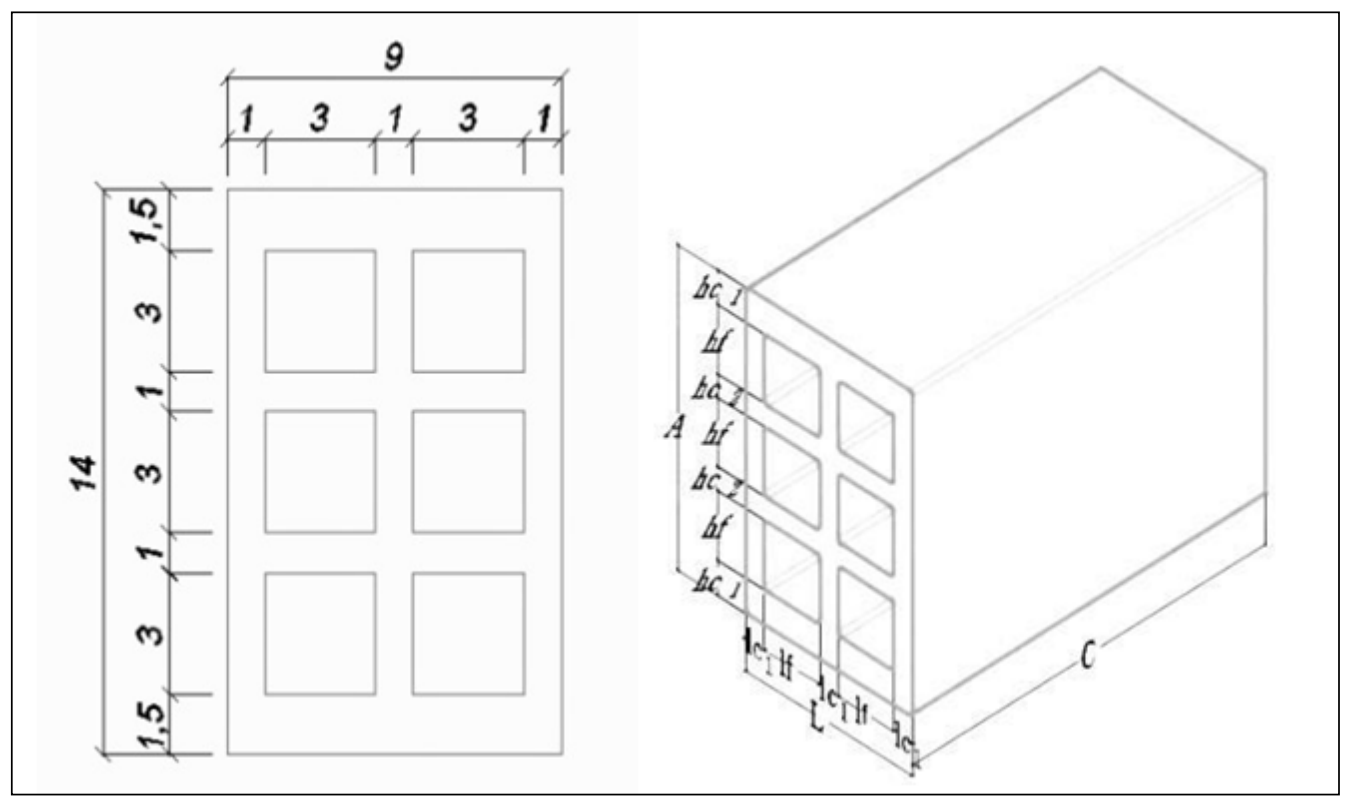

Fonte: Autores (2020).

Nas Figuras 02 e 03, está apresentado o projeto arquitetônico e corte da edificação. 
Figura 02 - Planta baixa do arquitetônico.

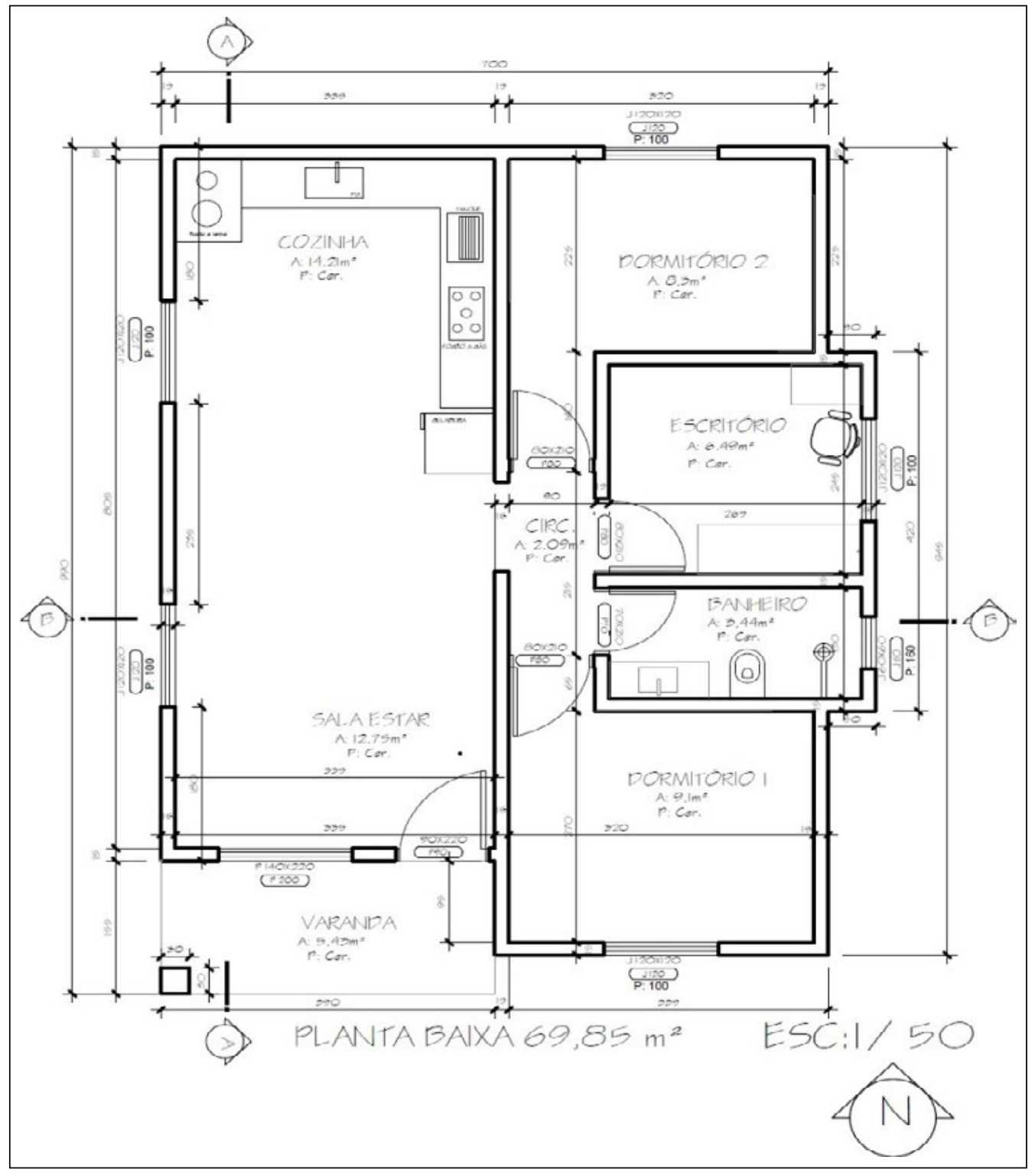

Fonte: Autores (2020). 
Figura 03 - Planta do corte (A-A)

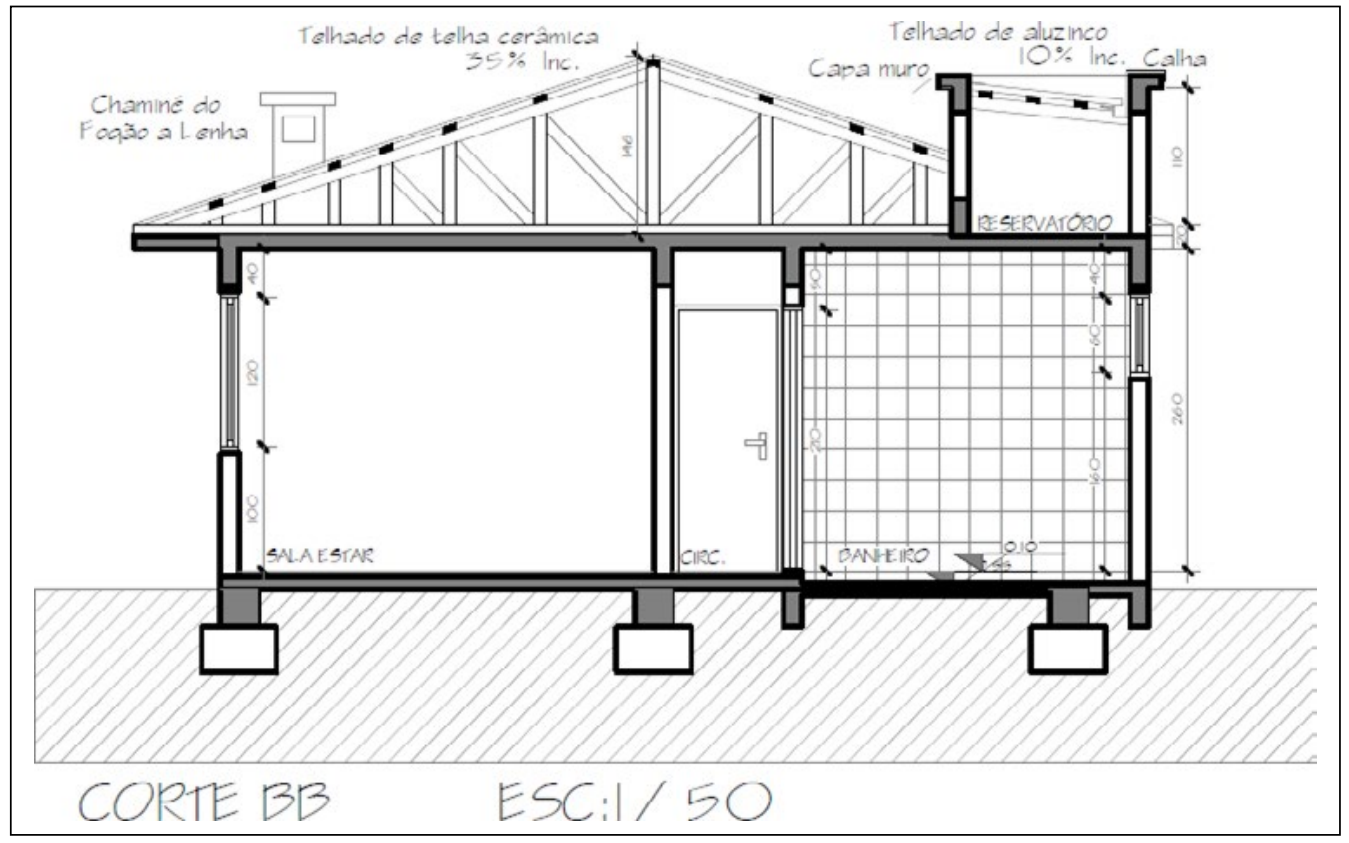

Fonte: Autores (2020).

\section{MEMORIAL DE CÁLCULO DO PROJETO A SER ADAPTADO}

\subsection{Transmitância térmica da parede}

Com posse dos dados elencados no Quadro 01, a seguir, poderemos descobrir a área de uma dada seção e a resistência térmica dela, conforme nos resultados a seguir:

Quadro 01 - Dados definidos para cálculo da transmitância

\begin{tabular}{|l|l|}
\hline e reboco & $2,5 \mathrm{~cm}$ \\
\hline e argamassa & $1,0 \mathrm{~cm}$ \\
\hline$\lambda$ reboco $/ \lambda$ argamassa & $1,5 \mathrm{~W}(\mathrm{~m} . \mathrm{K})$ \\
\hline$\lambda$ tijolo & $0,90 \mathrm{~W}(\mathrm{~m} . \mathrm{K})$ \\
\hline Dimensões tijolo & $9 \times 14 \times 19 \mathrm{~cm}$ \\
\hline
\end{tabular}

Fonte: Dos autores (2020).

A Seção "A" é composta pelo somatório da resistência térmica das camadas de: [reboco + argamassa + reboco]. 


$$
\begin{aligned}
& \mathrm{A}_{\mathrm{a}}=(0,01 \times 0,19)+(0,01 \times 0,15)=0,0034 \mathrm{~m}^{2} \\
& \mathrm{R}_{\mathrm{a}}=\frac{e_{\text {reboco }}}{\lambda_{\text {reboco }}}+\frac{e_{\text {argamassa }}}{\lambda_{\text {argamassa }}}+\frac{e_{\text {reboco }}}{\lambda_{\text {reboco }}}=0,1217 \frac{\mathrm{m}^{2} \cdot \mathrm{K}}{\mathrm{W}}
\end{aligned}
$$

A Seção "B" é composta pelo somatório da resistência térmica das camadas de: [reboco + tijolo + reboco].

$$
\begin{aligned}
& \mathrm{A}_{\mathrm{b}}=0,015 \times 0,19=0,0029 \mathrm{~m}^{2} \\
& \mathrm{R}_{\mathrm{b}}=\frac{e_{\text {reboco }}}{\lambda_{\text {reboco }}}+\frac{e_{\text {tijolo }}}{\lambda_{\text {tijolo }}}+\frac{e_{\text {reboco }}}{\lambda_{\text {reboco }}}=0,1435 \frac{\mathrm{m}^{2} . \mathrm{K}}{\mathrm{W}}
\end{aligned}
$$

A Seção "C" é composta pelo somatório da resistência térmica das camadas de: [reboco + tijolo + ar + tijolo + ar + tijolo + reboco $]$.

$$
\begin{aligned}
& \mathrm{A}_{\mathrm{c}}=0,03 \times 0,19=0,0057 \mathrm{~m}^{2} \\
& \mathrm{R}_{\mathrm{c}}=\frac{e_{\text {reboco }}}{\lambda_{\text {reboco }}}+\frac{e_{\text {parede tijolo }}}{\lambda_{\text {tijolo }}}+R_{\text {ar }}+\frac{e_{\text {parede tijolo }}}{\lambda_{\text {tijolo }}}+R_{\text {ar }}+\frac{e_{\text {parede tijolo }}}{\lambda_{\text {tijolo }}}+\frac{e_{\text {reboco }}}{\lambda_{\text {reboco }}}= \\
& \mathrm{R}_{\mathrm{c}}=0,3968 \frac{\mathrm{m}^{2} \cdot \mathrm{K}}{\mathrm{W}}
\end{aligned}
$$

Quadro 02 - Valores do fluxo horizontal

\begin{tabular}{|l|l|}
\hline Resistência térmica interna & $0,13 \frac{\mathrm{m}^{2} \cdot \mathrm{K}}{\mathrm{W}}$ \\
\hline Resistência térmica externa & $0,04 \frac{\mathrm{m}^{2} \cdot \mathrm{K}}{\mathrm{W}}$ \\
\hline
\end{tabular}

Fonte: Dos autores (2020).

Com propriedade das áreas e resistências de cada seção se torna viável o cálculo de resistência térmica da parede $\left(R_{t}\right)$. Com esse resultado, mais o acréscimo de valores de resistências térmicas internas e externas, conseguimos calcular a resistência total $\left(\mathrm{R}_{\mathrm{T}}\right)$ e com isso obtemos a transmitância térmica $(\mathrm{U})$. O cálculo se encontra melhor especificado a seguir:

Resistência térmica da parede:

$$
\mathrm{R}_{\mathrm{t}}=\frac{\mathrm{A}_{\mathrm{a}}}{\mathrm{A}_{\mathrm{a}}}+\frac{4 \times \mathrm{A}_{\mathrm{b}}}{\frac{4 \times \mathrm{A}_{\mathrm{b}}}{\mathrm{R}_{\mathrm{b}}}}+\frac{3 \times \mathrm{A}_{\mathrm{c}}}{3 \times \mathrm{A}_{\mathrm{c}}}=0,2120 \frac{\mathrm{m}^{2} \cdot \mathrm{K}}{\mathrm{W}}
$$

Resistência térmica total da parede:

$$
\mathrm{R}_{\mathrm{T}}-\mathrm{R}_{\mathrm{si}}+\mathrm{R}_{\mathrm{t}}+\mathrm{R}_{\mathrm{se}}=0,3820 \frac{\mathrm{m}^{2} \cdot \mathrm{K}}{\mathrm{W}}
$$

Transmitância térmica da parede:

$$
\mathrm{U}=\frac{1}{\mathrm{R}_{\mathrm{T}}}=2,62 \frac{\mathrm{W}}{\mathrm{m}^{2} \cdot \mathrm{K}}
$$




\section{Transmitância térmica do telhado}

O projeto motivo de estudo tem uma cobertura com camada de ar não ventilada. A variável da resistência térmica deriva da NBR 15220/2003 Resistencia térmica de câmaras de ar não ventiladas, com a largura muito maior que a espessura. A direção do fluxo de calor é descendente e as resistências são:

Quadro 03 - Resistências térmicas

\begin{tabular}{|l|l|}
\hline Resistência térmica interna & $0,17 \frac{m^{2} \cdot K}{W}$ \\
\hline Resistência térmica externa & $0,04 \frac{m^{2} . K}{W}$ \\
\hline
\end{tabular}

Fonte: Dos autores (2020).

Quadro 04 - Dados aplicados na lógica de cálculo

\begin{tabular}{|l|l|}
\hline$e_{\text {tellha de barro: }}$ & $1 \mathrm{~cm}$ \\
\hline $\mathrm{e}_{\text {forro de madeira }}$ & $1,5 \mathrm{~cm}$ \\
\hline$\lambda_{\text {barro }}$ & $0,70 \mathrm{~W} /(\mathrm{m} . \mathrm{K})$ \\
\hline$\lambda_{\text {madeira }}$ & $0,15 \mathrm{~W} /(\mathrm{m} . \mathrm{K})$ \\
\hline $\mathrm{R}_{\mathrm{ar}}$ & $0,21 \frac{\mathbf{m}^{2} \cdot \mathbf{K}}{\mathbf{W}}$ \\
\hline
\end{tabular}

Fonte: Dos autores (2020).

Resistência térmica do telhado:

$\mathrm{R}_{\mathrm{t}}=\frac{\mathrm{e}_{\text {barro }}}{\lambda_{\text {barro }}}+\mathrm{R}_{\mathrm{ar}}+\frac{\mathrm{e}_{\text {madeira }}}{\lambda_{\text {madeira }}}=0,3211 \frac{\mathrm{m}^{2} \cdot \mathrm{K}}{\mathrm{W}}$

Resistência térmica total da cobertura:

$\mathrm{R}_{\mathrm{T}}-\mathrm{R}_{\mathrm{si}}+\mathrm{R}_{\mathrm{t}}+\mathrm{R}_{\mathrm{se}}=0,5311 \frac{\mathrm{m}^{2} \mathrm{~K}}{\mathrm{~W}}$

Transmitância térmica da cobertura:

$$
\mathrm{U}=\frac{1}{\mathrm{R}_{\mathrm{T}}}=1,88 \frac{\mathrm{W}}{\mathrm{m}^{2} \cdot \mathrm{K}}
$$

Transmitância térmica das aberturas

Para este cálculo consideramos janela veneziana de madeira na cor pêssego com vidro simples $6 \mathrm{~mm}$. O fator solar usado para o vidro foi extraído da tabela disponibilizada nas notas de aula do professor. 
Quadro 05 - Resistências aplicadas na lógica de cálculo

\begin{tabular}{|l|l|}
\hline Resistência térmica interna & $0,13 \frac{\mathrm{m}^{2} \mathrm{~K}}{\mathrm{~W}}$ \\
\hline Resistência térmica externa & $0,04 \frac{\mathrm{m}^{2} \mathrm{~K}}{\mathrm{~W}}$ \\
\hline
\end{tabular}

Fonte: Dos autores (2020).

Com propriedade dos dados elencados ficou possível calcular a resistência térmica da esquadria. A resistência térmica do vidro e da veneziana de madeira equivalem ao $(\mathrm{Rt})$, na sequência são somadas as resistências interna e externas que derivam a resistência térmica total (RT), posteriormente podemos quantificar a transmitância térmica (U).

Quadro 06 - Dados empregados na lógica do cálculo

\begin{tabular}{|l|l|}
\hline $\mathrm{e}_{\text {vidro simples }}$ & $6 \mathrm{~mm}$ \\
\hline$\lambda_{\text {vidro simples }}$ & $1,0 \mathrm{~W} /(\mathrm{m} . \mathrm{K})$ \\
\hline FS & 0,83 \\
\hline
\end{tabular}

Fonte: Dos autores (2020).

Resistência térmica do vidro:

$\mathrm{R}_{\mathrm{t}}=\frac{\mathrm{e}_{\text {vidro simples }}}{\lambda_{\text {vidro simples }}}=0,006 \frac{\mathrm{m}^{2} . \mathrm{K}}{\mathrm{W}}$

Resistência térmica total do vidro:

$\mathrm{R}_{\mathrm{T}}=\mathrm{R}_{\mathrm{si}}+\mathrm{R}_{\mathrm{t}}+\mathrm{R}_{\mathrm{se}}=0,176 \frac{\mathrm{m}^{2} \cdot \mathrm{K}}{\mathrm{W}}$

Transmitância térmica do vidro:

$\mathrm{U}=\frac{1}{\mathrm{R}_{\mathrm{T}}}=5,681818182 \frac{\mathrm{W}}{\mathrm{m}^{2} \cdot \mathrm{K}}$

Quadro 07 - Dados da veneziana de madeira

\begin{tabular}{|l|l|}
\hline $\mathrm{e}_{\text {madeira }}$ & $2,5 \mathrm{~cm}$ \\
\hline$\lambda_{\text {madeira }}$ & $0,15 \mathrm{~W}(\mathrm{~m} . \mathrm{K})$ \\
\hline
\end{tabular}

Fonte: Dos autores (2020).

Resistência térmica da madeira:

$\mathrm{R}_{\mathrm{t}}=\frac{\mathrm{e}_{\text {madeira }}}{\lambda_{\text {madeira }}}=0,1667 \frac{\mathrm{m}^{2} \cdot \mathrm{K}}{\mathrm{W}}$ 
Resistência térmica total da madeira:

$$
\mathrm{R}_{\mathrm{T}}=\mathrm{R}_{\mathrm{si}}+\mathrm{R}_{\mathrm{t}}+\mathrm{R}_{\mathrm{se}}=0,3367 \frac{\mathrm{m}^{2} \cdot \mathrm{K}}{\mathrm{w}}
$$

Transmitância térmica da madeira:

$$
\mathrm{U}=\frac{1}{\mathrm{R}_{\mathrm{T}}}=2,97 \frac{\mathrm{W}}{\mathrm{m}^{2} \cdot \mathrm{K}}
$$

Primeiro cômodo - dormitório 01

O primeiro cômodo (dormitório 01) possui três paredes com dimensões de 3,20(sul) x 2,70(leste) x 0,95 (oeste) $\mathrm{m}$, com pé direito interno de 2,60 m. A parede norte possui uma esquadria de dimensões de $120 \times 120 \mathrm{~cm}$, as demais paredes são vedadas.

\section{Parede leste}

Índice de Absorvidade ( $\alpha$ ) e do Índice de Radiação Solar (I), estes valores foram obtidos das tabelas disponibilizadas nas aulas do professor, podemos definir a carga térmica que esta parede recebe. Com as paredes pintadas na cor pêssego, o índice de absorvidade é de 42,8 . O horário com maior incidência solar para esta parede são às $08 \mathrm{~h} 00 \mathrm{~min}$, o que corresponde ao índice de radiação solar de 715 Watts no mês de dezembro. Com estes dados, é calculado o fluxo térmico e o fluxo térmico total que atravessa o fechamento. Em seguida pode-se obter a carga total (CT) para a parede Sul.

$$
\begin{aligned}
& A_{\text {parede leste }}=7,02 \mathrm{~m}^{2} \\
& \mathrm{q}_{\text {fo parede leste }}=60,8409 \frac{\mathrm{W}}{\mathrm{m}^{2} \cdot \mathrm{K}} \\
& \mathrm{Q}_{\text {parede leste }}=427,1031 \mathrm{~W} \\
& \mathrm{CT}_{\text {parede leste }}=127,103 \mathrm{~W}
\end{aligned}
$$

Parede Sul

Índice de Absorvidade ( $\alpha$ ) e do Índice de Radiação Solar (I), estes valores foram obtidos das tabelas disponibilizadas nas notas de aula do professor, podemos definir a carga térmica que esta parede recebe. Com as paredes pintadas na cor pérola, o índice de absorvidade é de 42,8 . O horário com maior incidência solar para esta parede são às $17 \mathrm{~h} 00 \mathrm{~min}$, o que corresponde ao índice de radiação solar de 188 Watts. Com estes dados, descontando o valor da área da abertura é calculado o fluxo térmico e o fluxo térmico total que atravessa o fechamento.

Esta parede possui uma janela de 1,20 x 1,20 m. O fator solar do vidro simples é 0,83 . Na janela veneziana cor terracota utilizou-se o índice de absorvidade de 64,6. Deste modo foi necessário calcular fluxo térmico total do 
vidro e da veneziana de madeira. A partir da soma dos fluxos térmicos totais de cada materialidade foi possível obter a carga total (CT) para a parede Leste.

$$
\begin{aligned}
& A_{\text {parede sul }}=8,32 \mathrm{~m}^{2} \\
& q_{\text {fo parede sul }}=37,222 \frac{\mathrm{W}}{\mathrm{m}^{2} \cdot \mathrm{K}} \\
& \mathrm{Q}_{\text {parede sul }}=256,088 \mathrm{~W} \\
& A_{\text {vidro }}=0,72 \mathrm{~m}^{2} \\
& q_{A}=62,50 \frac{w}{m^{2}} \\
& \mathrm{q}_{\mathrm{s}}=156,04 \frac{\mathrm{w}}{\mathrm{m}^{2}} \\
& \mathrm{q}_{\text {FT vidro }}=218,54 \frac{\mathrm{w}}{\mathrm{II}^{2}} \\
& Q_{\text {vidro }}=A_{\text {vidro }} \times q_{\text {FT vidro }}=157,349 \mathrm{~W} \\
& \mathrm{~A}_{\text {madeira }}=0,72 \mathrm{~m}^{2} \\
& \mathrm{q}_{\mathrm{fo \text {madeira }}}=47,103 \frac{\mathrm{W}}{\mathrm{m}^{2} \cdot \mathrm{K}} \\
& \mathrm{Q}_{\text {madeira }}=33,914 \mathrm{~W} \\
& \mathrm{CT}_{\text {parede leste }}=447,3504 \mathrm{~W}
\end{aligned}
$$

Parede oeste

Índice de Absorvidade ( $\alpha$ ) e do Índice de Radiação Solar (I), estes valores foram obtidos das tabelas disponibilizadas nas aulas do professor, podemos definir a carga térmica que esta parede recebe. Com as paredes pintadas na cor pêssego, o índice de absorvidade é de 42,8 . O horário com maior incidência solar para esta parede são às $16 \mathrm{~h} 00 \mathrm{~min}$, o que corresponde ao índice de radiação solar de 715 Watts no mês de dezembro. Com estes dados, é calculado o fluxo térmico total que atravessa o fechamento. Em seguida pode-se obter a carga total (CT) para a parede Sul. 


$$
\begin{aligned}
& A_{\text {parede oeste }}=2,47 \mathrm{~m}^{2} \\
& \mathrm{q}_{\text {fo parede oeste }}=60,8409 \frac{\mathrm{W}}{\mathrm{m}^{2} \cdot \mathrm{K}} \\
& \mathrm{Q}_{\text {parede oeste }}=150,2770 \mathrm{~W} \\
& \mathrm{CT}_{\text {parede oeste }}=Q_{\text {parede oeste }}=150,2770 \mathrm{~W}
\end{aligned}
$$

\section{Cobertura}

A partir do Índice de Absorvidade ( $\alpha$ ) e do Índice de Radiação Solar (I), estes valores foram obtidos das tabelas disponibilizadas em norma, podemos definir a carga térmica que esta parede recebe. Com a cobertura de telha de barro, pintada na cor pérola, o índice de absorvidade utilizado é o de 33. O horário com maior incidência solar para a cobertura é às $12 \mathrm{~h} 00 \mathrm{~min}$, o que corresponde ao índice de radiação solar de 1134 Watts. Com estes dados, são calculados o fluxo térmico e o fluxo térmico total que atravessa o fechamento. Em seguida pode-se obter a carga total (CT) para a cobertura.

$$
\begin{aligned}
& \mathrm{A}_{\text {cobertura }}=8,64 \mathrm{~m}^{2} \\
& \mathrm{q}_{\text {fo cobertura }}=48,8952 \frac{\mathrm{W}}{\mathrm{m}^{2} \cdot \mathrm{K}} \\
& \mathrm{Q}_{\text {cobertura }}=422,4548 \mathrm{~W} \\
& \mathrm{CT}_{\text {cobertura }}=Q_{\text {fo cobertura }}=422,4548 \mathrm{~W} \\
& \text { Carga Térmica Total } \\
& \mathrm{CT}_{\text {Total }}=1447,1853 \mathrm{~W} \\
& \text { Ar Condicionado }
\end{aligned}
$$

Sabendo-se as cargas térmicas totais de cada ambiente, pode-se transformar de Watts

(W) para BTU/h e assim saber qual a demanda de ar condicionado para o cômodo calculado. A partir do cálculo da demanda acrescido de $500 \mathrm{BTU}$, valor este considerando duas pessoas por cômodo, encontra-se o ar condicionado com a potência disponível no mercado que possa suprir a necessidade de cada ambiente.

Ar Condicionado Calculado Dormitrrio 01 $=1447,1853=5437,80$ BTUs

Neste caso o ar condicionado disponível no mercado para atender esta demanda é o de 7000 BTUs. 
Segundo cômodo - sala de estar

O segundo cômodo (sala de estar) possui dimensões de 8,05(oeste) $\mathrm{x}$ 3,35 (norte) $\times 3,35$ (sul) $\mathrm{m}$, com pé direito interno de 2,6 m. A parede norte não possui janelas, a oeste possui duas janelas, sendo elas de dimensões de $120 \mathrm{x}$ $120 \mathrm{~cm}$, e a parede sul possui uma porta $(0,90 \times 2,20 \mathrm{~m})$ e uma janela $(1,40 \times 2,20$ $\mathrm{m})$. As demais paredes são vedadas.

\section{Parede Oeste}

A partir do Índice de Absorvidade $(\alpha)$ e do Índice de Radiação Solar (I), estes valores foram obtidos das tabelas disponibilizadas nas notas de aula do professor, podemos definir a carga térmica que esta parede recebe. Com as paredes pintadas na cor pêssego, o índice de absorvidade é de 42,8. O horário com maior incidência solar para esta parede são às 16:00 horas, o que corresponde ao índice de radiação solar de 715 Watts. Com estes dados, é calculado o fluxo térmico e o fluxo térmico total que atravessa o fechamento. Em seguida pode-se obter a carga total (CT) para a parede Sul.

Falar que são duas aberturas de 1,2 $\times 1,2 \mathrm{~m}$.

$$
\begin{aligned}
& A_{\text {parede oeste }}=20,93 \mathrm{~m}^{2} \\
& \mathrm{q}_{\text {fo parede oeste }}=60,8409 \frac{\mathrm{W}}{\mathrm{m}^{2} \cdot \mathrm{K}} \\
& Q_{\text {parede oeste }}=1098,1781 \mathrm{~W} \\
& \mathrm{~A}_{\text {vidro }}=1,44 \mathrm{~m}^{2} \\
& \mathrm{q}_{\mathrm{A}}=62,50 \frac{\mathrm{w}}{\mathrm{II}^{2}} \\
& \mathrm{q}_{\mathrm{s}}=593,45 \frac{\mathrm{w}}{\mathrm{m}^{2}} \\
& \mathrm{q}_{\mathrm{FT} \text { vidro }}=655,95 \frac{\mathrm{w}}{\mathrm{In}}{ }^{2} \\
& Q_{\text {vidro }}=A_{\text {vidro }} \times \mathrm{q}_{\mathrm{FT} \text { vidro }}=911,568 \mathrm{~W}
\end{aligned}
$$




$$
\begin{aligned}
& \mathrm{A}_{\text {madeira }}=1,44 \mathrm{~m}^{2} \\
& \mathrm{q}_{\text {fo madeira }}=87,5513 \frac{\mathrm{W}}{\mathrm{m}^{2} \cdot \mathrm{K}} \\
& \mathrm{Q}_{\text {madeira }}=126,074 \mathrm{~W} \\
& \mathrm{CT}_{\text {parede oeste }}=2168,8200 \mathrm{~W}
\end{aligned}
$$

\section{Parede Sul}

A partir do Índice de Absorvidade $(\alpha)$ e do Índice de Radiação Solar (I), estes valores foram obtidos das tabelas disponibilizadas nas notas de aula do professor, podemos definir a carga térmica que esta parede recebe. Com as paredes pintadas na cor pêssego, o índice de absorvidade é de 42,8 . O horário com maior incidência solar para esta parede são às 16:00 horas, 0 que corresponde ao índice de radiação solar de 715 Watts. Com estes dados, descontando o valor da área da abertura é calculado o fluxo térmico e o fluxo térmico total que atravessa o fechamento.

Esta parede possui uma janela de 1,40 x 2,20 m e uma porta de $0,90 \mathrm{x}$ $2,20 \mathrm{~m}$. O fator solar do vidro simples é 0,83 . Na janela veneziana cor terracota utilizou-se o índice de absorvidade de 64,6. Deste modo foi necessário calcular fluxo térmico total do vidro e da veneziana de madeira. A partir da soma dos fluxos térmicos totais de cada materialidade foi possível obter a carga total (CT) para a parede Leste.

$$
\begin{aligned}
& A_{\text {parede sul }}=8,71 \mathrm{~m}^{2} \\
& q_{\text {fo parede sul }}=60,8409 \frac{\mathrm{W}}{\mathrm{m}^{2} \cdot \mathrm{K}} \\
& Q_{\text {parede sul }}=222,0693 \mathrm{~W} \\
& A_{\text {vidro }}=1,54 \mathrm{~m}^{2} \\
& q_{A}=62,5 \frac{\mathrm{w}}{\mathrm{m}^{2}} \\
& q_{\mathrm{S}}=593,450 \frac{\mathrm{W}}{\mathrm{m}^{2}} \\
& q_{\text {FT vidro }}=655,95 \frac{\mathrm{w}}{\mathrm{m}^{2}} \\
& Q_{\text {vidro }}=1010,1630 \mathrm{~W}
\end{aligned}
$$




$$
\begin{aligned}
& A_{\text {madeira }}-3,52 \mathrm{~m}^{2} \\
& \mathrm{q}_{\text {fo madeira }}=87,5513 \frac{\mathrm{W}}{\mathrm{m}^{2} \cdot \mathrm{K}} \\
& \mathrm{Q}_{\text {madeira }}=308,1805 \mathrm{~W} \\
& \mathrm{Cl}_{\text {parede sul }}=1540,4128 \mathrm{~W}
\end{aligned}
$$

Parede norte

Índice de Absorvidade ( $\alpha$ ) e do Índice de Radiação Solar (I), estes valores foram obtidos das tabelas disponibilizadas nas aulas do professor, podemos definir a carga térmica que esta parede recebe. Com as paredes pintadas na cor pêssego, o índice de absorvidade é de 42,8 . O horário com maior incidência solar para esta parede são às $12 \mathrm{~h} 00 \mathrm{mi}$, o que corresponde ao índice de radiação solar de 179 Watts no mês de dezembro. Com estes dados, são calculados o fluxo térmico e o fluxo térmico total que atravessa o fechamento. Em seguida pode-se obter a carga total (CT) para a parede Sul.

$$
\begin{aligned}
& A_{\text {parede norte }}=8,71 \mathrm{~m}^{2} \\
& \mathrm{q}_{\text {fo parede norte }}=36,8187 \frac{\mathrm{W}}{\mathrm{m}^{2} \cdot \mathrm{K}} \\
& \mathrm{Q}_{\text {parede norte }}=320,6907 \mathrm{~W} \\
& \mathrm{CT}_{\text {parede norte }}=Q_{\text {parede }}=320,6907 \mathrm{~W}
\end{aligned}
$$

\section{Cobertura}

A partir do Índice de Absorvidade $(\alpha)$ e do Índice de Radiação Solar (I), estes valores foram obtidos das tabelas disponibilizadas nas notas de aula do professor, podemos definir a carga térmica que esta parede recebe. Com a cobertura de telha de barro pintada na cor pérola, o índice de absorvidade utilizado é 33. O horário com maior incidência solar para a cobertura é às $12 \mathrm{~h} 00 \mathrm{~min}$, o que corresponde ao índice de radiação solar de 1134 Watts. Com estes dados, é calculado o fluxo térmico e o fluxo térmico total que atravessa o fechamento. Em seguida pode-se obter a carga total (CT) para a cobertura. 


$$
\begin{aligned}
& \mathrm{A}_{\text {cobertura }}=26,968 \mathrm{~m}^{2} \\
& \mathrm{q}_{\text {fo cobertura }}=48,8952 \frac{\mathrm{W}}{\mathrm{m}^{2} \cdot \mathrm{K}} \\
& \mathrm{Q}_{\text {cobertura }}=1318,5821 \mathrm{~W} \\
& \mathrm{CT}_{\text {cobertura }}=\mathrm{Q}_{\text {fo cobertura }}=1318,5821 \mathrm{~W} \\
& \text { Carga Térmica Total } \\
& \mathrm{CT}_{\text {Total }}=5348,5056 \mathrm{~W} \\
& \text { Ar Condicionado }
\end{aligned}
$$

Sabendo-se as cargas térmicas totais de cada ambiente, pode-se transformar de Watts

(W) para BTU/h e assim saber qual a demanda de ar condicionado para o cômodo calculado. A partir do cálculo da demanda acrescido de 500 BTU, valor este considerando duas pessoas por cômodo, encontra-se o ar condicionado com a potência disponível no mercado que possa suprir a necessidade de cada ambiente.

Ar Condicionado Calculado Sala de Estar $=5348,5056=18749,10$ BTUs. Neste caso o ar condicionado disponível no mercado para atender esta demanda é o de20.000 BTUs.

Terceiro cômodo - dormitório 02

O terceiro cômodo (dormitório 02) possui duas paredes com dimensões de $3,20 \times 2,25$, com pé direito interno de $2,60 \mathrm{~m}$. A parede norte possui uma esquadria de dimensões de $120 \times 120 \mathrm{~cm}$, a outra parede é vedada.

Parede leste

Índice de Absorvidade $(\alpha)$ e do Índice de Radiação Solar (I), estes valores foram obtidos das tabelas disponibilizadas nas aulas do professor, podemos definir a carga térmica que esta parede recebe. Com as paredes pintadas na cor pêssego, o índice de absorvidade é de 42,8 . O horário com maior incidência solar para esta parede são às 8:00 horas, o que corresponde ao índice de radiação solar de 715 Watts no mês de dezembro. Com estes dados, é calculado o fluxo térmico e o fluxo térmico total que atravessa o fechamento. Em seguida pode-se obter a carga total (CT) para a parede Sul. 


$$
\begin{aligned}
& A_{\text {parede leste }}=5,85 \mathrm{~m}^{2} \\
& \mathrm{q}_{\text {fo parede leste }}=60,8409 \frac{\mathrm{W}}{\mathrm{m}^{2} \cdot \mathrm{K}} \\
& \mathrm{Q}_{\text {parede leste }}=355,9192 \mathrm{~W} \\
& \mathrm{CT}_{\text {parede leste }}=\mathrm{Q}_{\text {parede leste }}=355,9192 \mathrm{~W}
\end{aligned}
$$

\section{Parede Norte}

Índice de Absorvidade ( $\alpha$ ) e do Índice de Radiação Solar (I), estes valores foram obtidos das tabelas disponibilizadas nas notas de aula do professor, podemos definir a carga térmica que esta parede recebe. Com as paredes pintadas na cor pérola, o índice de absorvidade é de 42,8 . O horário com maior incidência solar para esta parede são às 12:00 horas, o que corresponde ao índice de radiação solar de 179 Watts. Com estes dados, descontando o valor da área da abertura é calculado o fluxo térmico e o fluxo térmico total que atravessa o fechamento.

Esta parede possui uma janela de 1,20 x 1,20 m. O fator solar do vidro simples é 0,83 . Na janela veneziana cor terracota utilizou-se o índice de absorvidade de 64,6. Deste modo foi necessário calcular fluxo térmico total do vidro e da veneziana de madeira. A partir da soma dos fluxos térmicos totais de cada materialidade foi possível obter a carga total (CT) para a parede Leste.

$$
\begin{aligned}
& A_{\text {parede norte }}=8,32 \mathrm{~m}^{2} \\
& \mathrm{q}_{\text {fo parede norte }}=36,819 \frac{\mathrm{W}}{\mathrm{m}^{2} \cdot \mathrm{K}} \\
& Q_{\text {parede norte }}=253,3125 \mathrm{~W} \\
& \mathrm{~A}_{\text {vidro }}=0,72 \mathrm{~m}^{2} \\
& \mathrm{q}_{\mathrm{A}}=62,50 \frac{\mathrm{w}}{\mathrm{m}^{2}} \\
& \mathrm{q}_{\mathrm{S}}=148,570 \frac{\mathrm{w}}{\mathrm{m}^{2}} \\
& \mathrm{q}_{\mathrm{FT} \text { vidro }}=211,07 \frac{\mathrm{w}}{\mathrm{m}^{2}} \\
& Q_{\text {vidro }}=\mathrm{A}_{\text {vidro }} \times \mathrm{q}_{\mathrm{FT} \text { vidro }}=151,97 \mathrm{~W}
\end{aligned}
$$




$$
\begin{aligned}
& A_{\text {madeira }}=0,72 \mathrm{ml}^{2} \\
& \mathrm{q}_{\text {fo madeira }}=46,412 \frac{\mathrm{W}}{\mathrm{m}^{2} \cdot \mathrm{K}} \\
& \mathrm{Q}_{\text {madeira }}=33,417 \mathrm{~W} \\
& \mathrm{CT}_{\text {parede leste }}=438,6995 \mathrm{~W} .
\end{aligned}
$$

\section{Cobertura}

A partir do Índice de Absorvidade ( $\alpha$ ) e do Índice de Radiação Solar (I), estes valores foram obtidos das tabelas disponibilizadas nas notas de aula do professor, podemos definir a

carga térmica que esta parede recebe. Com a cobertura de telha de barro, pintada na cor pérola, o índice de absorvidade utilizado é o de 33 . O horário com maior incidência solar para a cobertura é às $12 \mathrm{~h} 00 \mathrm{~min}$, o que corresponde ao índice de radiação solar de 1134 Watts. Com estes dados, é calculado o fluxo térmico e o fluxo térmico total que atravessa o fechamento. Em seguida pode-se obter a carga total (CT) para a cobertura.

$$
\begin{aligned}
& A_{\text {cobertura }}=8,32 \mathrm{~m}^{2} \\
& \mathrm{q}_{\text {fo cobertura }}=48,8952 \frac{\mathrm{W}}{\mathrm{m}^{2} \cdot \mathrm{K}} \\
& \mathrm{Q}_{\text {cobertura }}=406,8083 \mathrm{~W} \\
& \mathrm{CT}_{\text {cobertura }}=\mathrm{Q}_{\text {fo cobertura }}=406,8083 \mathrm{~W} \\
& \text { Carga Térmica Total } \\
& \mathrm{CT}_{\text {Total }}=1201,4271 \mathrm{~W} \\
& \text { Ar Condicionado }
\end{aligned}
$$

Sabendo-se as cargas térmicas totais de cada ambiente, pode-se transformar de Watts (W) para BTU/h e assim saber qual a demanda de ar condicionado para o cômodo calculado. A partir do cálculo da demanda acrescido de 500 BTU, valor este considerando duas pessoas por cômodo, encontra-se o ar condicionado com a potência disponível no mercado que possa suprir a necessidade de cada ambiente. Ar Condicionado Calculado $\mathrm{Dormitório} 02_{2}=$ $1201,4271=4599,27$ BTUs. Neste caso o ar condicionado disponível no mercado para atender esta demanda é o de 7000 BTUs. 


\section{APRESENTAÇÃO DO PROJETO COM AS MELHORIAS PROPOSTAS}

Por motivos construtivos as paredes externas foram consideradas com espessura de 18,5 cm, de tijolo cerâmico de seis furos. A parede é rebocada nas duas faces e pintadas de cor pêssego. A primeira medida adotada na parte interna foi a do poliestireno estrudado de espessura de $3 \mathrm{~cm}$ e placas de gesso acartonado de $1,5 \mathrm{~cm}$. Já na cobertura, que é de forro de madeira (pinus), espessura de 1,5 $\mathrm{cm}$ e o telhado de telhas de barro, pintadas na cor de pérola e espessura compatível a $1,0 \mathrm{~cm}$. O forro recebeu um acréscimo de 3,0 de poliestireno estrudado e uma manta de lá de vidro $(5 \mathrm{~cm})$ sob as telhas.

Memorial de cálculo do projeto com as melhorias propostas

Com as alterações sofridas as transmitâncias térmicas da parede e da cobertura sofreram alterações que estão propostas abaixo:

Transmitância térmica da parede:

$$
\mathrm{U}=0,75 \frac{\mathrm{W}}{\mathrm{m}^{2} \cdot \mathrm{K}}
$$

Transmitância térmica da cobertura:

$$
\begin{aligned}
& \mathrm{U}=0,40 \frac{\mathrm{W}}{\mathrm{m}^{2} \cdot \mathrm{K}} \\
& \text { PRIMEIRO CÔMODO - DORMITÓRIO } 01
\end{aligned}
$$

\section{Parede leste}

Todos os dados, referentes a esta parede se encontram melhor descritos, no capítulo anterior.

$$
\begin{aligned}
& A_{\text {parede leste }}=2,70 \times 2,60=7,02 \mathrm{~m}^{2} \\
& \mathrm{q}_{\text {fo parede leste }}=46,2595 \frac{\mathrm{W}}{\mathrm{m}^{2} \cdot \mathrm{K}} \\
& \mathrm{Q}_{\text {parede leste }}=324,7414 \mathrm{~W} \\
& \mathrm{CT}_{\text {parede sul }}=Q_{\text {parede sul }}=324,7414 \mathrm{~W}
\end{aligned}
$$

\section{Parede Sul}

Os dados utilizados no cálculo desta parede se encontram especificados no capítulo anterior. 
$A_{\text {parede sul }}=8,32 \mathrm{~m}^{2}$

$\mathrm{q}_{\text {fo parede sul }}=28,301 \frac{\mathrm{W}}{\mathrm{m}^{2} \cdot \mathrm{K}}$

$\mathrm{Q}_{\text {parede sul }}=194,712 \mathrm{~W}$

O vidro e a veneziana continuam com os mesmos valores calculados anteriormente.

$\mathrm{CT}_{\text {parede sul }}=385,9751 \mathrm{~W}$

Parede oeste

Todos os dados, referentes a esta parede se encontram melhor descritos, no capítulo anterior.

$A_{\text {parede oeste }}=2,47 \mathrm{~m}^{2}$

$q_{\text {fo parede oeste }}=46,2595 \frac{\mathrm{W}}{\mathrm{m}^{2} \cdot \mathrm{K}}$

$\mathrm{Q}_{\text {parede oeste }}=114,2608 \mathrm{~W}$

$\mathrm{CT}_{\text {parede oeste }}=\mathrm{Q}_{\text {parede sul }}=114,2608 \mathrm{~W}$

Cobertura

Todos os dados, referentes a esta parede se encontram melhor descritos, no capítulo anterior.

$$
\begin{aligned}
& \mathrm{A}_{\text {cobertura }}=8,64 \mathrm{~m}^{2} \\
& \mathrm{q}_{\text {fo cobertura }}=10,3902 \frac{\mathrm{W}}{\mathrm{m}^{2} \cdot \mathrm{K}} \\
& \mathrm{Q}_{\text {cobertura }}=89,7710 \mathrm{~W} \\
& \mathrm{CT}_{\text {cobertura }}=\mathrm{Q}_{\text {fo cobertura }}=89,7710 \mathrm{~W} \\
& \text { Carga Térmica Total } \\
& \mathrm{CT}_{\text {Total }}=914,7483 \mathrm{~W}
\end{aligned}
$$

Ar Condicionado

Sabendo-se as cargas térmicas totais de cada ambiente, pode-se transformar de Watts 
(W) para BTU/h e assim saber qual a demanda de ar condicionado para o cômodo calculado. A partir do cálculo da demanda acrescido de 500 BTU, valor este considerando duas pessoas por cômodo, encontra-se o ar condicionado com a potência disponível no mercado que possa suprir a necessidade de cada ambiente.

Ar Condicionado Calculado ${ }_{\text {Dormitório 01 }}=914,7483=7000$ BTUs

Neste caso o ar condicionado disponível no mercado para atender esta demanda é o de 7000 BTUs.

Segundo cômodo - sala de estar Parede Oeste

Todos os dados utilizados no cálculo se encontram melhor especificados no capítulo anterior. Falar que são duas aberturas de 1,2x1,2m.

$$
\begin{aligned}
& A_{\text {parede oeste }}=20,93 \mathrm{~m}^{2} \\
& \mathrm{q}_{\text {fo parede oeste }}=46,2595 \frac{\mathrm{W}}{\mathrm{m}^{2} \cdot \mathrm{K}} \\
& Q_{\text {parede oeste }}=834,9831 \mathrm{~W} \\
& \text { O vidro e a veneziana por não sofrerem nenhuma melhoria continuam }
\end{aligned}
$$
com os mesmos valores calculados anteriormente.

$$
\mathrm{CT}_{\text {Total }}=1905,6250 \mathrm{~W}
$$

\section{Parede Sul}

Todos os dados utilizados no cálculo se encontram melhor especificados no capítulo anterior.

$$
\begin{aligned}
& A_{\text {parede sul }}=8,71 \mathrm{~m}^{2} \\
& \mathrm{q}_{\text {fo parede sul }}=46,259 \frac{\mathrm{W}}{\mathrm{m}^{2} \cdot \mathrm{K}} \\
& Q_{\text {parede sul }}=169,130 \mathrm{~W} \\
& O \text { vidro e a veneziana por não sofrerem nenhuma melhoria continuam }
\end{aligned}
$$
com os mesmos valores calculados anteriormente.

$$
\mathrm{CT}_{\text {parede norte }}=1487,4733 \mathrm{~W}
$$

Parede norte

Todos os dados utilizados no cálculo se encontram melhor especificados no capítulo anterior. 


$$
\begin{aligned}
& A_{\text {parede norte }}=8,71 \mathrm{~m}^{2} \\
& \mathrm{q}_{\text {fo parede norte }}=28,0414 \frac{\mathrm{W}}{\mathrm{m}^{2} \cdot \mathrm{K}} \\
& \mathrm{Q}_{\text {parede norte }}=244,2406 \mathrm{~W} \\
& \mathrm{CT}_{\text {parede norte }}=Q_{\text {parede sul }}=244,2406 \mathrm{~W}
\end{aligned}
$$

Cobertura

Todos os dados utilizados no cálculo se encontram melhor especificados no capítulo anterior.

$$
\begin{aligned}
& \mathrm{A}_{\text {cobertura }}=26,968 \mathrm{~m}^{2} \\
& \mathrm{q}_{\text {fo cobertura }}=10,3902 \frac{\mathrm{W}}{\mathrm{m}^{2} \cdot \mathrm{K}} \\
& \mathrm{Q}_{\text {cobertura }}=280,1966 \mathrm{~W} \\
& \mathrm{CT}_{\text {cobertura }}=\mathrm{Q}_{\text {fo cobertura }}=280,1966 \mathrm{~W} \\
& \text { Carga Térmica Total } \\
& \mathrm{CT}_{\text {Total }}=3918,9338 \mathrm{~W} \\
& \text { Ar Condicionado }
\end{aligned}
$$

Sabendo-se as cargas térmicas totais de cada ambiente, pode-se transformar de Watts

(W) para BTU/h e assim saber qual a demanda de ar condicionado para o cômodo calculado. A partir do cálculo da demanda acrescido de 500 BTU, valor este considerando duas pessoas por cômodo, encontra-se o ar condicionado com a potência disponível no mercado que possa suprir a necessidade de cada ambiente.

Ar Condicionado Calculado sala de estar $=3918,9338=13871,40$ BTUs

Neste caso o ar condicionado disponível no mercado para atender esta demanda é o de

18.000 BTUs.

Terceiro cômodo - dormitório 02

O terceiro cômodo (dormitório 02) possui duas paredes com dimensões de $3,20 \times 2,25$, com pé direito interno de $2,60 \mathrm{~m}$. A parede norte possui uma esquadria de dimensões de $120 \times 120 \mathrm{~cm}$, a outra parede é vedada. 


\section{Parede leste}

Todos os dados utilizados no cálculo se encontram melhor especificados no capítulo anterior.

$$
\begin{aligned}
& \mathrm{A}_{\text {parede leste }}=5,85 \mathrm{~m}^{2} \\
& \mathrm{q}_{\text {fo parede leste }}=46,3369 \frac{\mathrm{W}}{\mathrm{m}^{2} \cdot \mathrm{K}} \\
& \mathrm{Q}_{\text {parede leste }}=271,0710 \mathrm{~W} \\
& \mathrm{CT}_{\text {parede leste }}=\mathrm{Q}_{\text {parede leste }}=271,0710 \mathrm{~W}
\end{aligned}
$$

Parede Norte

Todos os dados utilizados no cálculo se encontram melhor especificados no capítulo anterior.

$$
\begin{aligned}
& A_{\text {parede norte }}=8,32 \mathrm{~m}^{2} \\
& \mathrm{q}_{\text {fo parede norte }}=28,041 \frac{\mathrm{W}}{\mathrm{m}^{2} \cdot \mathrm{K}} \\
& \mathrm{Q}_{\text {parede norte }}=192,925 \mathrm{~W}
\end{aligned}
$$

O vidro e a veneziana por não sofrerem nenhuma melhoria continuam com os mesmos valores calculados anteriormente.

$$
\mathrm{CT}_{\text {parede norte }}=378,3119 \mathrm{~W}
$$

\section{Cobertura}

Os dados das variáveis utilizadas no cálculo estão especificadas no capítulo anterior.

$$
\begin{aligned}
& \mathrm{A}_{\text {cobertura }}=8,32 \mathrm{~m}^{2} \\
& \mathrm{q}_{\text {fo cobertura }}=10,3902 \frac{\mathrm{W}}{\mathrm{m}^{2} \cdot \mathrm{K}} \\
& \mathrm{Q}_{\text {cobertura }}=86,4461 \mathrm{~W} \\
& \mathrm{CT}_{\text {cobertura }}=\mathrm{Q}_{\text {fo cobertura }}=86,4461 \mathrm{~W} \\
& \text { Carga Térmica Total } \\
& \mathrm{CT}_{\text {Total }}=735,8290 \mathrm{~W}
\end{aligned}
$$




\section{Ar Condicionado}

Sabendo-se as cargas térmicas totais de cada ambiente, pode-se transformar de Watts

(W) para BTU/h e assim saber qual a demanda de ar condicionado para o cômodo calculado. A partir do cálculo da demanda acrescido de 500 BTU, valor este considerando duas pessoas por cômodo, encontra-se o ar condicionado com a potência disponível no mercado que possa suprir a necessidade de cada ambiente.

Ar Condicionado Calculado dormitório 02 $=735,8290=3010,65$ BTUs

Neste caso o ar condicionado disponível no mercado para atender esta demanda é o de 7000 BTUs.

\section{CONCLUSÃO}

Concluímos, portanto que com esse estudo, quando a edificação tem um projeto térmico, o ganho em economia em longo prazo é bem significativo, em certos casos em até curto período de tempo. Observamos, também que o estudo de materiais e técnicas que vedam o calor são fundamentais, para um projeto de bom conforto térmico e consciência ambiental. A edificação readaptada minimizou a carga térmica, gerando uma diferença significativa de potência requerida. Também é perceptível ver que as alterações contribuíram para minoração da transmitância térmica, ou seja, a demanda por ar condicionado teve uma redução em todos os cômodos. Isso torna a edificação mais sustentável, sem deixar de atender as normas de conforto térmico. Ao final, concluímos que a utilização de projeto e técnicas construtivas, somadas a adoção de materialidades e análise térmica é possível otimizar o conforto térmico de uma edificação Minha Casa Minha Vida (MCMV).

\section{REFERÊNCIAS}

ASHRAE, ASHRAE Handbook - Fundamentals, American Soceity of Heating, Refrigerating, and Air Conditioning Engineers, Atlanta, 2005.

ASSOCIAÇÃO BRASILEIRA DE NORMAS TÉCNICAS. NBR 15220 - Norma de desempenho térmico de edificações. Rio de Janeiro: ABNT, 2003.

ASSOCIAÇÃO BRASILEIRA DE NORMAS TÉCNICAS. NBR 15575 - Norma de desempenho para edificações habitacionais. Rio de Janeiro: ABNT, 2015.

ASSOCIAÇÃO BRASILEIRA DE NORMAS TÉCNICAS. NBR 16401 - Norma de ar condicionado . Rio de Janeiro: ABNT, 2008.

Desempenho Térmico de Edificações - Laboratório de Eficiência Energética de Edificações da UNIVERSIDADE FEDERAL DE SANTA CATARINA. Disponível em:<www.ceap.br/material/MAT 25022013164631.pdf > Acessado em: 19 de outubro de 2016. 